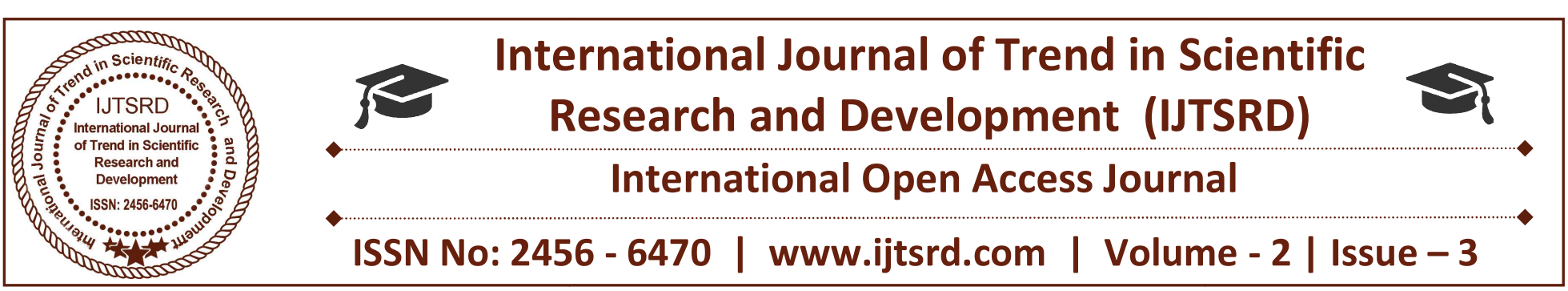

\title{
Voice controlled notice board using bluetooth module
}

\author{
Priyanka. T. Ranit \\ Prof. Ram Meghe College of Engineering \& Management, \\ Badnera, Amravati, Maharashtra, India
}

\begin{abstract}
Main concept behind Voice operated Electronic notice board using display is to show messages and to control them by using our own voice. We have already seen GSM based Electronic Notice board, however speech controlled Notice board has additional advantage of ease of use. User has to give voice command in his/her own voice to control the messages displayed on electronic notice board. Voice recognition is done in the Android application. User has to install this Android application in his/her smart phone or tablet.
\end{abstract} objective of this work is to develop a smart notice

Bluetooth wireless technology is a popular technique in the field communication, and it is one of the fastest growing fields in the wireless technologies. Bluetooth technology handles the wireless part of the communication channel; it is used in this system to transmit and receive data wirelessly between devices.

User needs to install this application. A computer system used for this purpose is called a speech synthesizer, and can be implemented in software or hardware products. An inbuilt Google voice recognition app is used in this system.

\section{LITERATURE REVIEW}

This system deals with the implementation of voicebased system by using Bluetooth with the help of android application. The communication is made possible by using Bluetooth module. The main

board which works in a well-organized manner with respect to date and time which will help the user to easily keep in track of the notice board every day and each time he uses the system and to convey the information more effectively. [2]

This system focuses on GSM (Global System for Mobile) technology to design a digital notice board, "Wireless Notice Board using GSM System" is wireless module which send message wirelessly with the help of GSM module. This means that users or registered persons may be able to send messages from anywhere and this message is displayed on the LCD display. [3]

The main objective of this system is to develop a wireless e-notice board that displays message sent from the user and to design a simple, easy to install, user friendly system, which can receive and display notice in a particular manner with respect to date and time which will help the user to easily keep the track of notice board every day and each time he uses the system. GSM and Wi-Fi are the wireless technology used in this system they use Wi-Fi module for data transmission. The main disadvantage of this system is using Wi-Fi is the network failure. [4] 


\section{SYSTEM DESCRIPTION \& WORKING}

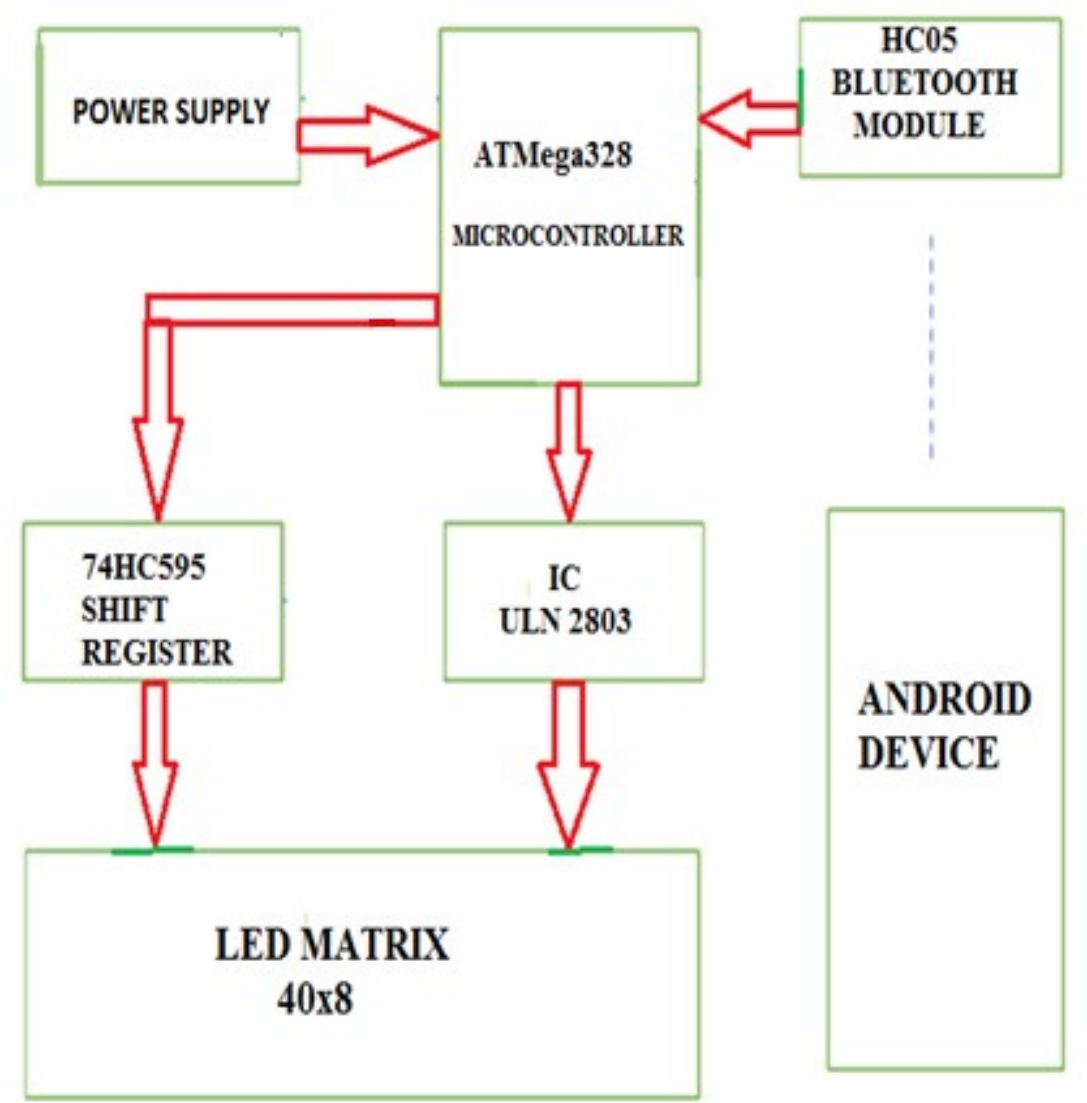

Figure 3.1.Block Diagram Of Voice Controlled Notice Board

\section{WORKING:}

There exists a need of electronic notice board that enables efficient way to the user for displaying notice. By considering increasing compactness of electronic systems, there is a need of embedding two or more systems together. This system is an implementation of the idea of wireless communication between a mobile phone and an ATmega328 controller. In this system work, as seen in fig3.1 we are designing a system which consists of display unit, and android device using wireless technology. The display unit consists of LED display that can be interfaced with ATmega328 microcontroller. Bluetooth is an open wireless protocol for exchanging data over short distances from mobile devices, creating Personal Area Networks (PANs). It was originally conceived as a wireless alternative to RS232 data cables. It can connect several devices, overcoming problems of synchronization. Bluetooth will receive the signals sent by the Android application device (mobile phone), and then send these signals to the microcontroller. In order to implement this system, we need to use an Android application that is capable of performing the following Functions:

$>$ Convert voice data to text.

$>$ Send this text over to microcontroller via Bluetooth for displaying on notice board.

In the past we have seen a GSM based notice board which is time consuming and difficult to operate. We are designing a notice board which displays the message by our voice command and it is connected by the android application with the help of Bluetooth module. 


\section{HARDWARE DESCRIPTION}

\subsection{POWER SUPPLY:}

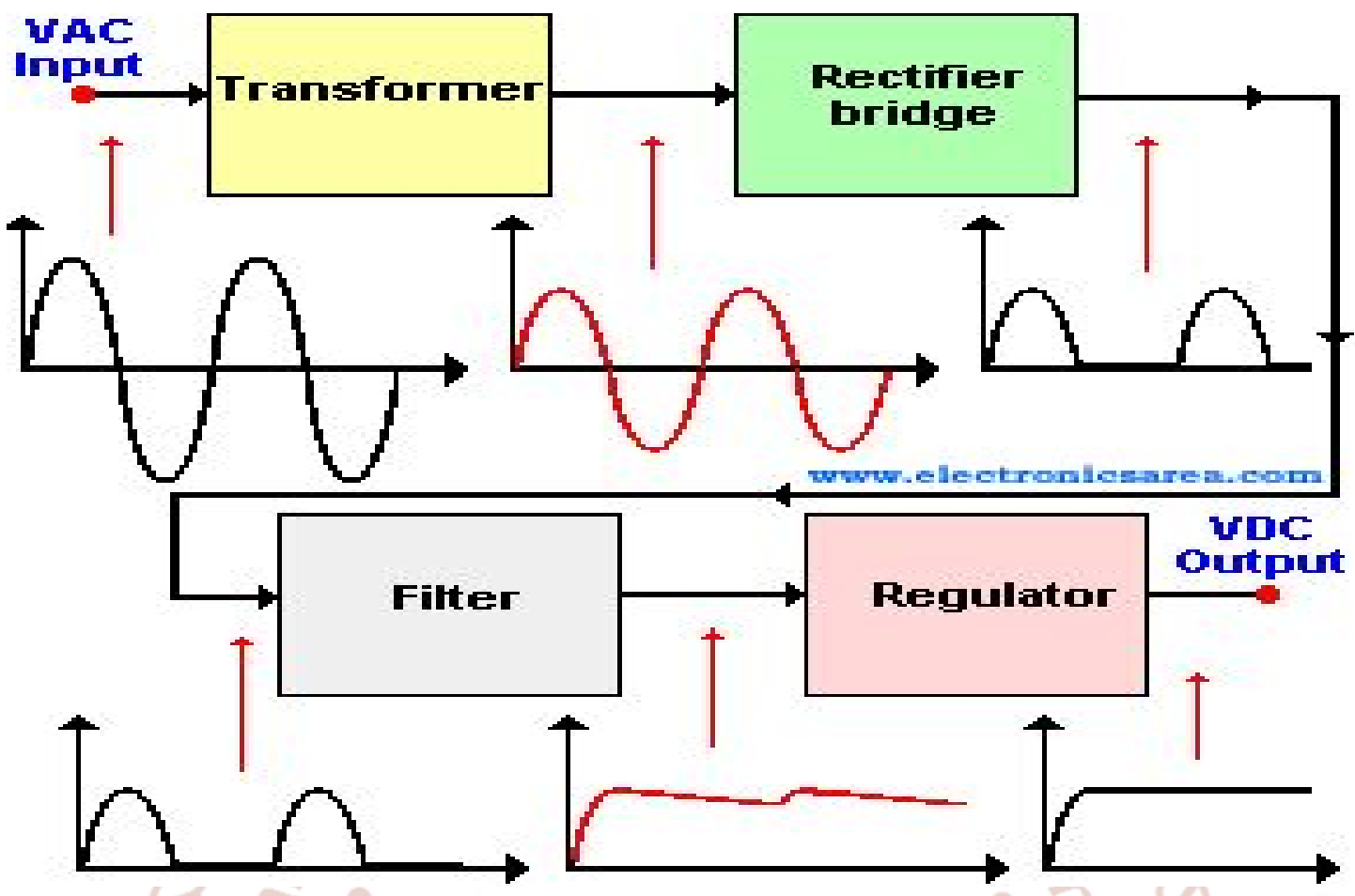

Fig4.1.Block Diagram of Power Supply

\section{Transformer:}

The electrical transformer receives on the primary winding an $\mathrm{AC}$ voltage and delivers on the secondary winding a different $\mathrm{AC}$ voltage (a lower one). This AC output voltage must be according to the DC voltage we want to obtain at the end. For example: If we want to obtain a final DC voltage of 12 volts, the secondary windings of the transformer must have an $\mathrm{AC}$ voltage no less of 9 volts.

The peak value on the secondary winding of the transformer will be $\mathrm{Vp}=1.41 \times 9=12.69$ volts. Even thought this value is very close to the one we wanted to get, it is not recommended because we need to take into account that the voltage drops at different stages (blocks) of the power supply. In this case, we can choose a transformer with a 12 volts AC secondary winding. With this AC voltage, we can get a peak voltage of: $\mathrm{Vp}=12 \times 1.41=16.92$ volts.

\section{Rectifier:}

The output of transformer is given as an input to the rectifier block. The rectifier transforms the secondary winding $\mathrm{AC}$ voltage into a pulsating DC voltage. . In our case, we use a $1 / 2$ wave rectifier, which eliminates the negative part of the wave an half wave rectifier conducts only during the positive half cycle of the $\mathrm{AC}$ input.

\section{Filter:}

The filter, formed by one or more capacitors, flattens or smoothes the previous wave eliminating the alternating current (AC) component delivered by the rectifier. These capacitors are charged to the maximum voltage value that the rectifier can deliver, and they are discharged when the pulsating signal disappears.

\section{Voltage regulator:}

The voltage regulator receives the signal from the filter and delivers a constant voltage (let's say 12 DC volts) regardless of the variations on the load or the voltage supply The dc signals are further given to the regulator that maintains the output of the power supply at a constant level. 


\subsection{HC-05 BLUETOOTH MODULE:}
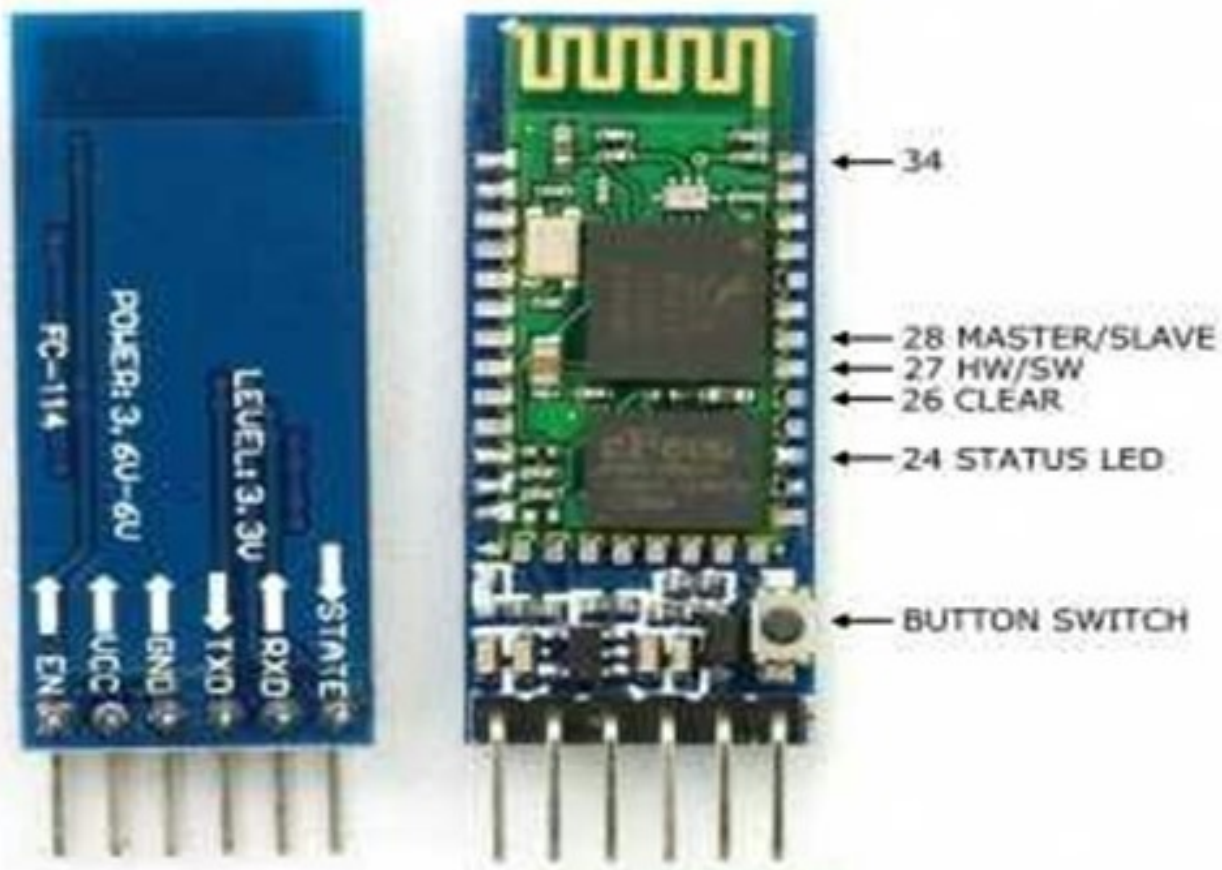

Fig4.2. HC-05 Bluetooth Module

$\mathrm{HC}-05$ module is an easy to use Bluetooth SPP (Serial Port Protocol) module, designed for transparent wireless serial connection setup. Serial port Bluetooth module is fully qualified Bluetooth V2.0+EDR (Enhanced Data Rate) 3Mbps Modulation with complete $2.4 \mathrm{GHz}$ radio transceiver and baseband. It is used for the transmission of data from android device to microcontroller.

\section{PIN DISCRIPTION:}

The HC-05 Bluetooth Module has 6pins. They are as follows:

\section{ENABLE:}

When enable is pulled LOW, the module is disabled which means the module will not turn on and it fails to co4mmunicate. When enable is left open or connected to $3.3 \mathrm{~V}$, the module is enabled i.e. the module remains on and communication also takes place.

\section{Vec:}

Supply Voltage $3.3 \mathrm{~V}$ to $5 \mathrm{~V}$.

GND:

Ground pin.

\section{TXD \& RXD:}

These two pins acts as an UART interface for communication.

\section{STATE:}

It acts as a status indicator. When the module is not connected to 10 paired with any other Bluetooth device, signal goes Low. At this low state, the led flash continuously which denotes that the module is not paired with other device. When this module is connected to/paired with any other Bluetooth device, the signal goes High. At this high state, the led blinks with a constant delay say for example $2 \mathrm{~s}$ delay which indicates that the module is paired.

\section{BUTTON SWITCH:}

This is used to switch the module into AT command mode. To enable AT command mode, press the button switch for a second. With the help of AT commands, the user can change the parameters of this module but only when the module is not paired with any other BT device. If the module is connected to any other Bluetooth device, it starts to communicate with that device and fails to work in AT command mode. 
International Journal of Trend in Scientific Research and Development (IJTSRD) ISSN: 2456-6470

\subsection{ATmega328:}

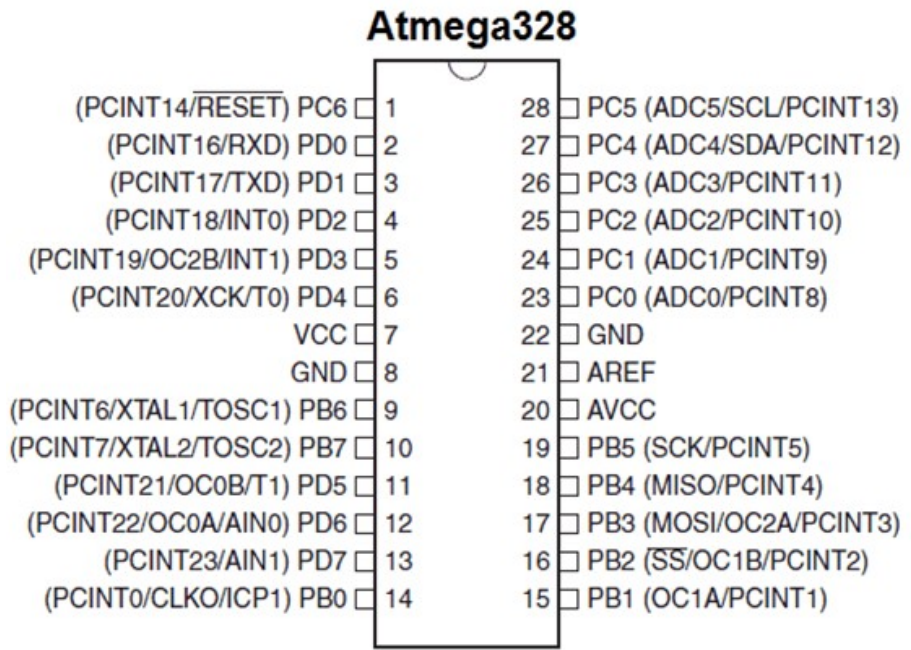

Fig4.3 Pin Diagram of ATmega328

- It is a 28 pin IC.

- We can design our system by using other controllers also but by using Atmega328 the size of the system can be reduced to great extent.

- The cost of the system also gets reduced.

- The input from android is sent over the microcontroller via Bluetooth for displaying it on notice board.

- In this system pin no. $2 \& 3$ are used for the transmission and reception purpose.

\subsection{ANDROID DEVICE:}

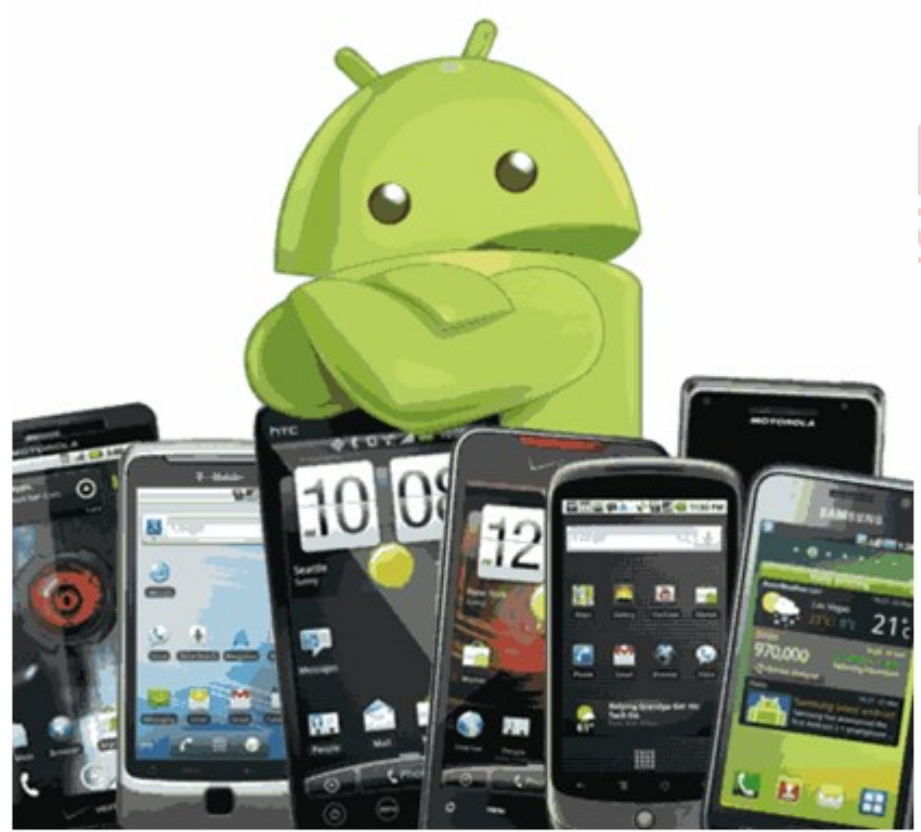

Fig 4.4 Android Device
- Android is a mobile operating system developed by Google which is used by several smart phones and tablets.

- For giving the data in the form of speech we can either use Google speech recognition app which is almost inbuilt in every android device.

- Otherwise we can use speech to text converter app.

\subsection{LED DISPLAY:}

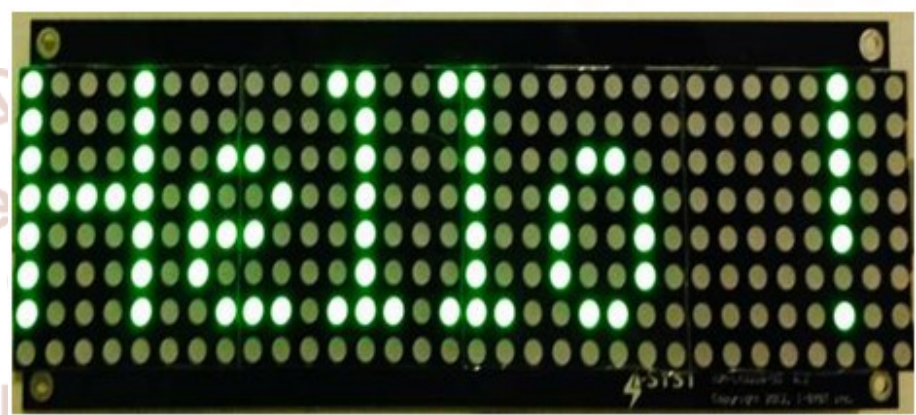

Fig.4.5.LED Display

- A 40x8 LED display is used in this system.

- The size of the display can be varied by adjusting the LED metrics.

- nThe LED will send the data in the form characters i.e. it will take one space then data and then \#.

- Whenever we are giving the input, it gives the first character as * then data i.e. matter and \#.

- \# indicates end up of data.

\subsection{IC ULN2803:}

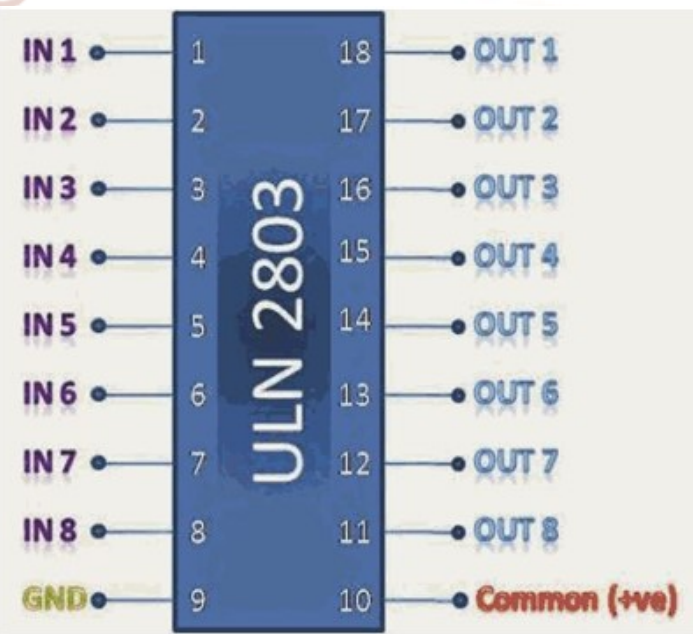

Fig4.6.Pin Diagram Of IC ULN2803 
- The IC (ULN2803) is a driver IC.

- All the LED's are connected to the port 2 of the driver IC.

- It is used to give the brightness to the LED.

\subsection{IC 74HC595:}

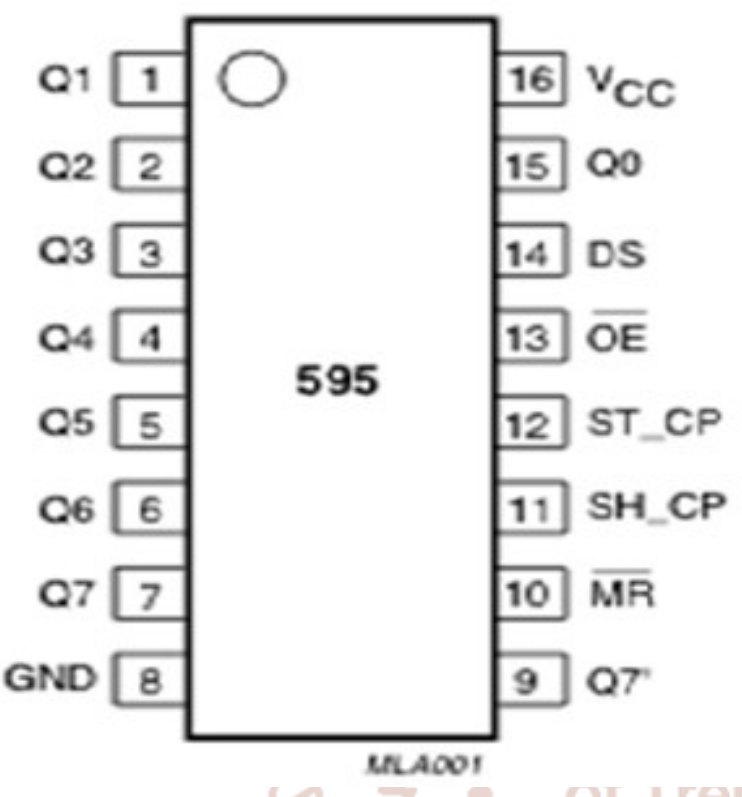

Fig4.7.Pin Diagram of IC 74HC595

- Instead of LCD we are using a rolling characters LED display.

- Therefore by using the shift register(74HC595) the function of shifting characters is

\section{Carried out.}

- The LCD display can display only 16 characters on a single line, but instead if we use the LED scrolling display we can display up to 50 characters on a single line.

\section{ADVANTAGES, DISADVANTAGES APPLICATIONS}

\subsection{ADVANTAGES:}

- Helpful for handicapped people.

- Low cost.

- User friendly \& Easy installation.

- Less power consumption.
- It overcomes all the demerits of previous system such as wastage of paper, time and labor.

\subsection{DISADVANTAGES:}

- Range is limited to 10 meters.

- Compared to GSM module max signal rate is low i.e. $1 \mathrm{Mb} / \mathrm{s}$.

\subsection{APPLICATIONS:}

- It can be used in colleges, schools, bus stands and railway stations.

- Industries where we can control machines by just saying instructions.

- Military applications.

- It can be used in malls \& highways for advertisement purpose.

\section{FUTURE SCOPE}

Temperature display during periods wherein no message buffers are empty is one such theoretical improvement that is well possible. Another very interesting and significant improvement would be to accommodate multiple receiver MODEMS at different positions in the geographical area carrying duplicate SIM cards. Multilingual display can be another added variation in the system. The Bluetooth printing has been implemented successfully with android phone and outputs have been verified. Future work focuses on implementation of Wi-Fi printing.

\section{CONCLUSION}

We are concluding that, by introducing the concept of wireless technology in the field of communication we can make our communication more efficient and faster, with greater efficiency we can display the \& messages with less errors and maintenance. We have presented an approach of using the Bluetooth technology for mobile printing from a palm handheld and explained the application for the purpose of printing from a mobile device. 